\title{
Características Histológicas de la Pulpa Dental de Ratones de 4 y 12 Semanas
}

\author{
Histological Features of Dental Pulp in 4 and 12 Week-old Mice
}

\author{
Mario Alejandro Ortiz Salazar \& Liliana Salazar Monsalve*
}

ORTIZ, S. M. A. \& SALAZAR, M. L. Características histológicas de la pulpa dental de ratones de 4 y 12 semanas. Int. J. Odontostomat., 8(2):159-164, 2014.

RESUMEN: En condiciones fisiológicas, el órgano dentinopulpar no se encuentra asociado a procesos de remodelación y resorción. Los hallazgos histológicos muestran que la pulpa presenta características especiales como tejido conectivo que le confieren su capacidad de adaptación. El propósito del presente estudio fue evaluar las características histológicas de la pulpa dental de ratones de la cepa Albino Suizo de 4 y 12 semanas de edad. Ocho hemimandíbulas de ratones albino suizo de 4 y 12 semanas de edad fueron utilizados. Las hemimandíbulas fueron fijadas en Paraformaldehído al 4\%, sometidas a decalcificación con EDTA al $5 \%$ durante 10 días a $4^{\circ} \mathrm{C}$ e incluidas en Paraplast $囚$. Se obtuvieron secciones de $5 \mu \mathrm{m}$ que fueron teñidos con H\&E, Tricrómico de Masson e Impregnación Argéntica. Las características histológicas de la pulpa revelaron dos zonas principales en ambos grupos de edad: la zona periférica pulpar conformada por un conjunto de células compatibles con odontoblastos y una zona pobre en células, y otra zona central pulpar, rica en fibroblastos y vasos sanguíneos. Las diferencias entre los grupos de edad se vieron en la expresión de matriz fibrilar de colágeno. Se encontraron semejanzas en la presencia de fibras reticulares entre los grupos. La pulpa dentaria en ratones Albino Suizo mostró una división en dos zonas: central y periférica, con grupos poblacionales bien definidos. La mayor diferencia por edad estuvo en la cantidad de colágeno.

PALABRAS CLAVE: pulpa dentaria, odontoblastos, colágeno.

\section{INTRODUCCIÓN}

El órgano dentinopulpar está conformado por un conjunto de tejidos de origen ectomesenquimatoso diferenciados durante el proceso de odontogénesis del germen dental. Estos tejidos se derivan de la papila dental y permiten en el adulto la formación de dos tejidos plenamente identificables, la dentina y la pulpa dental, quines constituyen una unidad biológica desde el punto de vista estructural, embriológico y funcional. Esta ubicación especial le brinda a este tejido conectivo algunas características especiales (Okiji, 2002; Goldberg \& Lasfargues, 1995).

Este tejido conectivo esta formado por una amplia red de células formadoras de matriz altamente especializadas como fibroblastos pulpares (Goldberg \& Smith, 2004) y odontoblastos (Arana-
Chavez \& Massa, 2004), células indiferenciadas (Renard et al., 2007) y células inmunocompetentes (Angelova et al., 2004). Estas células establecen relaciones complejas a través de los componentes fibrilar y no fibrilar de la matriz extracelular, presentando una arquitectura celular bien definida en regiones (Goldberg \& Smith). Como todos los tejidos conectivos, el proceso de envejecimiento pulpar se asocia a cambios en las proporciones entre células y matriz, la composición de la matriz fibrilar y no fibrilar, capacidad de respuesta celular, vascularización, entre otros (Schofield \& Weightman, 1978). El objetivo del presente estudio fue evaluar las características histológicas de la pulpa dental de ratones de la cepa Albino Suizo de 4 y 12 semanas de edad.

Odontólogo, Maestría en Ciencias Básicas Médicas. Especialista en Ortodoncia, Departamento de Morfología, Universidad del Valle, Campus San Fernando, Cali, Colombia.

* Fisioterapeuta, Maestría en Morfología. Profesor Asociado, Departamento de Morfología, Universidad del Valle, Campus San Fernando, Cali, Colombia.

Proyecto financiado por Vicerrectoría de Investigaciones, Universidad del Valle, Cali, Colombia. 


\section{MATERIAL Y MÉTODO}

En este estudio fueron usados ratones machos de la cepa Albino Suizo de 4 y 12 semanas (4 de cada uno) provenientes del Bioterio de la facultad de Salud de la Universidad del Valle. Todos los procedimientos fueron aprobados por el Comité de ética en Investigación Animal de la facultad de Salud de la Universidad del Valle (Acta No. 043, 10.09.06). A la edad de evaluación (4 y 12 semanas respectivamente) los ratones fueron sometidos a eutanasia intraperitoneal con Pentobarbital sódico, seguido de perfusión cardiaca con Paraformaldehido al $4 \%$. Las muestras obtenidas consistieron en 8 hemimandíbulas sometidas a fijación por inmersión en paraformaldehido al $4 \%$ a $24^{\circ} \mathrm{C}$ durante 48 horas. Posteriormente fueron lavadas con solución fisiológica a pH 7,4 durante una hora y sometidas a protocolo de decalcificación con EDTA al 5\% durante 10 días a $4^{\circ} \mathrm{C}$. Las muestras se sometieron a deshidratación en gradiente ascendente de alcoholes, aclaramiento en Xilol e inclusión en Paraplast $₫$, para luego ser cortadas en sentido mesio-distal en un micrótomo hasta obtener muestras de $5 \mu \mathrm{m}$ de grosor. Todas las muestras fueron sometidas a protocolos Hematoxilina - Eosina (H\&E), Tricrómica de Masson e Impregnación Argéntica.
Las muestras fueron evaluadas bajo microscopio de luz para evaluar la estructura normal de la pulpa de ratón Albino Suizo. Histotopográficamente la pulpa fue dividida en dos regiones según Pinzón et al. (1967) para mamíferos menores: Región central de la pulpa y Región periférica de la pulpa.

\section{RESULTADOS}

La pulpa dental de los ratones corresponde a un tejido conectivo laxo altamente celular, con presencia de vasos sanguíneos en su región central. En ella se reconoce una clara organización histo-arquitectónica asociada a las diferencias en los componentes celulares y de matriz extracelular. Gracias a estas diferencias, la pulpa se puede dividir en dos regiones: la zona periférica y la zona central de la pulpa (Fig. 1A y 1B).

En la zona Periférica de la pulpa, la cual se encuentra en contacto con la dentina, se pudo observar la presencia de alta densidad celular (Fig. 1). Las células de esta región son células cilíndricas o de morfología poligonal, mononucleares, polarizadas y con núcleo de ubicación distal a la unión dentinal (Fig. 1) características que se corresponden con la descripción tradicional

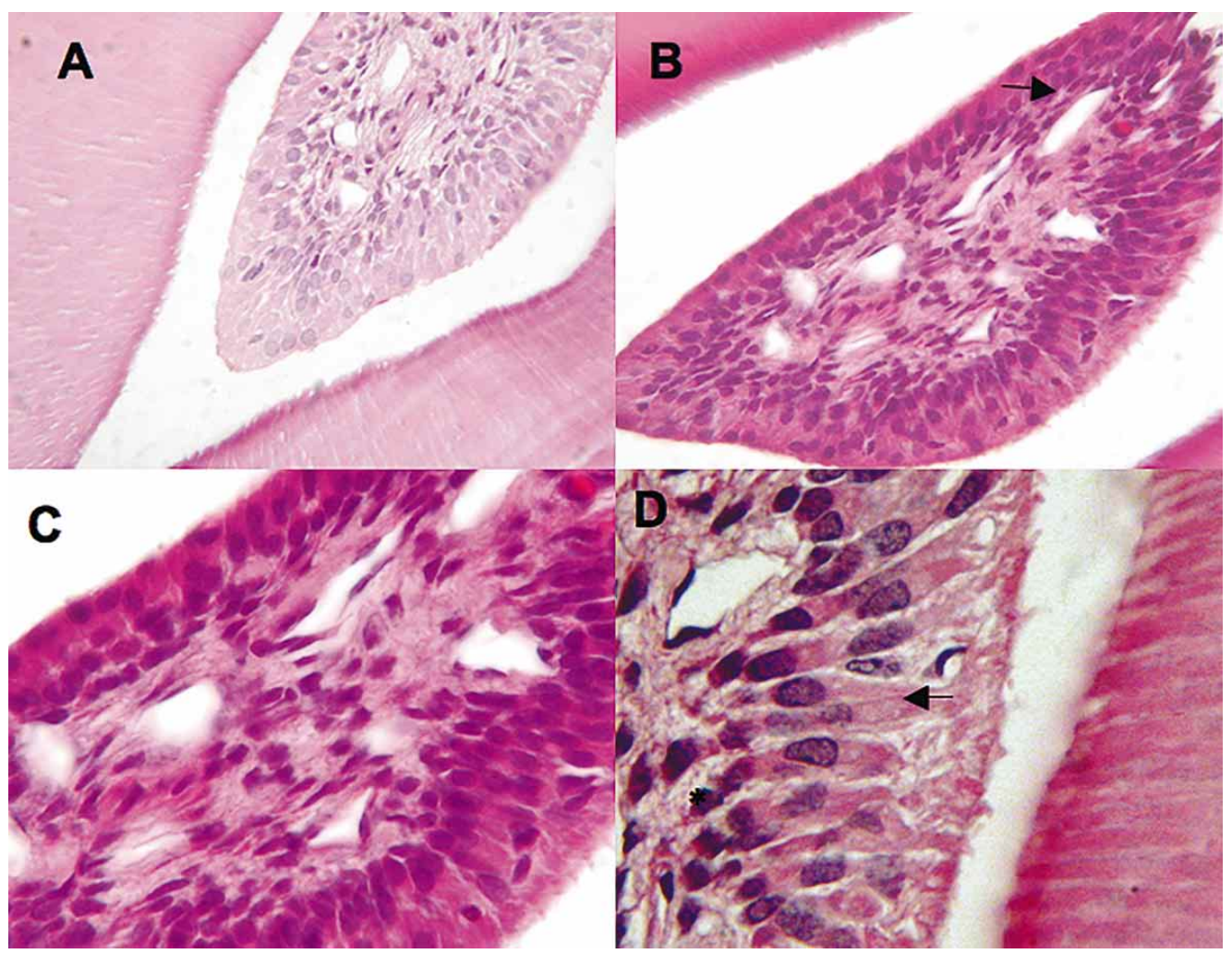

Fig. 1. Microfotografías de Cortes Histológicos de Molares de ratón teñidos con HematoxilinaEosina. En A y B se pueden identificar las diferencias en densidad celular a nivel de las regiones central y periférica de la pulpa. En C se puede observar un acercamiento de la zona. En D se puede ver la zona periférica. Nótese las características morfológicas de los odontoblastos y su organización en empalizada. El asterisco $\left({ }^{*}\right)$ muestra células redondeadas de la región subodontoblástica Aumento original de $4 \mathrm{X}$ en $A, 10 X$ en $B$ y $40 X$ en $C$ y $100 \times$ en $D$. 
de los odontoblastos. En la región adyacente a la predentina, se pueden observar proyecciones citoplasmáticas de estas células que ingresan a la dentina, compatibles con las prolongaciones odontoblásticas (Figs. 2C, D y E). En la capa subodontoblástica, es posible identificar la presencia de una pequeña cantidad de células redondeadas, con un núcleo ovalado y una elevada proporción núcleo-radio (Fig. 2). Estas células son poco abundantes y aparentan estar en contacto próximo a los odontoblastos, aunque es posible identificar entre algunas de ellas espacios intercelulares amplios. Por su ubicación y características morfológicas son compatibles con células madre mesenquimatosas de la pulpa dental.

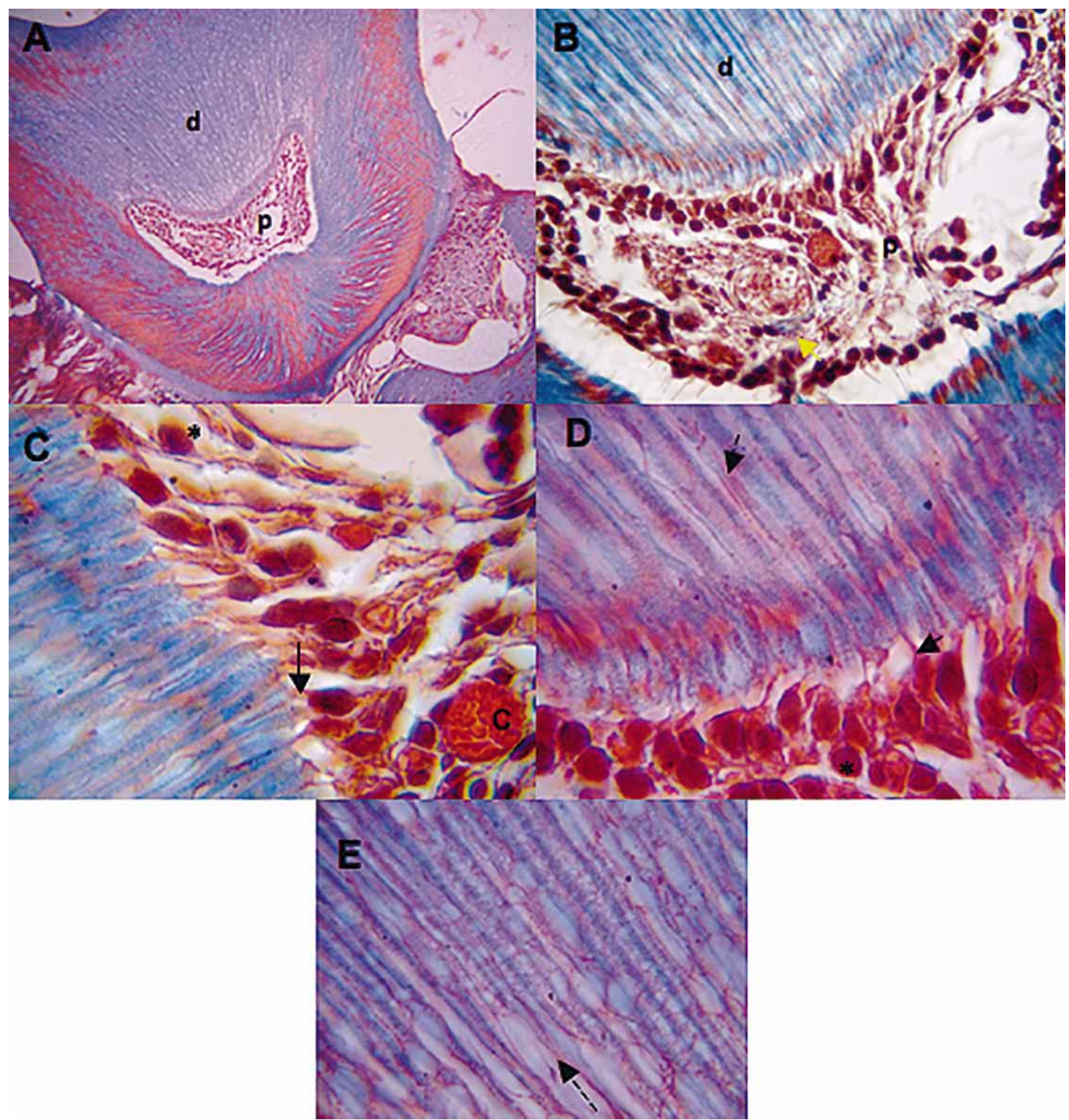

Fig. 2. Microfotografías de Cortes Histológicos de Molares de ratón teñidos con Tricrómico de Masson. En A se observa la distribución entre dentina (d) y pulpa (p) en el órgano dentino pulpar. En B se observa la organización a nivel de las regiones central y periférica de la pulpa. Observe señalado con la flecha amarilla la presencia de fibras de colágeno. En C y D se identifica la morfología odontoblástica, la presencia de la prolongación odontoblástica (Flecha continua) a nivel de la pulpa y su origen celular. Adicionalmente se observa un vaso sanguíneo subodontoblástico (c). El asterisco $\left(^{*}\right)$ muestra células redondeadas de la región subodontoblástica. La prolongación odontoblástica es mucho más notoria en la macrofotografía $D$ (flecha discontinua), en $E$ se puede ver con mayor detalle la proyección dentinal de la prolongación odontoblástica (Flecha discontinua). Aumento original de 4X en A, 10X en B y 100X en C, D y E. 
El componente de matriz extracelular fibrilar a nivel de esta región es escaso. No se observan fibras de colágeno entre los odontoblastos en las tinciones con Tricrómica de Masson (Fig. 2B). Este hallazgo es congruente tanto para ratones de 4 semanas como para los de 12 semanas. Al evaluar con la técnica de Gomori la presencia de Fibras Reticulares se puede observar la presencia de un plexo reticular a nivel de la región odontoblástica que da sostén a los odontoblastos, tanto en ratones de 4 como de 12 semanas. La zona central de la pulpa se encuentra alejada del límite dentinal y está recubierta en su totalidad por la región periférica (Fig. 1). Esta región se caracteriza por presentar una disminución en la densidad celular en comparación con la región periférica. Adicionalmente, presenta un aumento de espacio intercelular y de matriz extracelular, brindándole las características de un tejido conectivo laxo convencional. Las células más abundantes de esta región presentan morfología fusiforme con un núcleo ovalado, bien definido con cromatina gruesa, dispersa, y un citoplasma eosinófilo de difícil identificación en tinciones de H\&E (Fig. 1C y 1D). Al evaluarse usando la tinción Tricrómica de Masson, puede detallarse mejor el citoplasma de estas células identificando su morfología fusiforme y estrellada (Fig. 2C). Esta descripción coincide con las características de un fibroblasto. Adicionalmente es posible identificar células con núcleo redondeado y proporción elevada núcleo/citoplasma. El espacio intercelular entre estas células está lleno de matriz que presenta composición variable en las diferentes edades estudiadas en el ratón. En la edad de 4 semanas, la presencia de fibras de colágeno fue pobre. Sin embargo, en la medida en que aumenta la edad y madura el tejido conectivo (12 semanas), la expresión de colágeno aumenta, de manera coherente con los cambios etáreos que sufre el tejido conectivo. En la región central de la pulpa es posible identificar la presencia de vasos sanguíneos alrededor de los cuales se estructura toda la trama de células y fibras constitutivas de la pulpa dental.

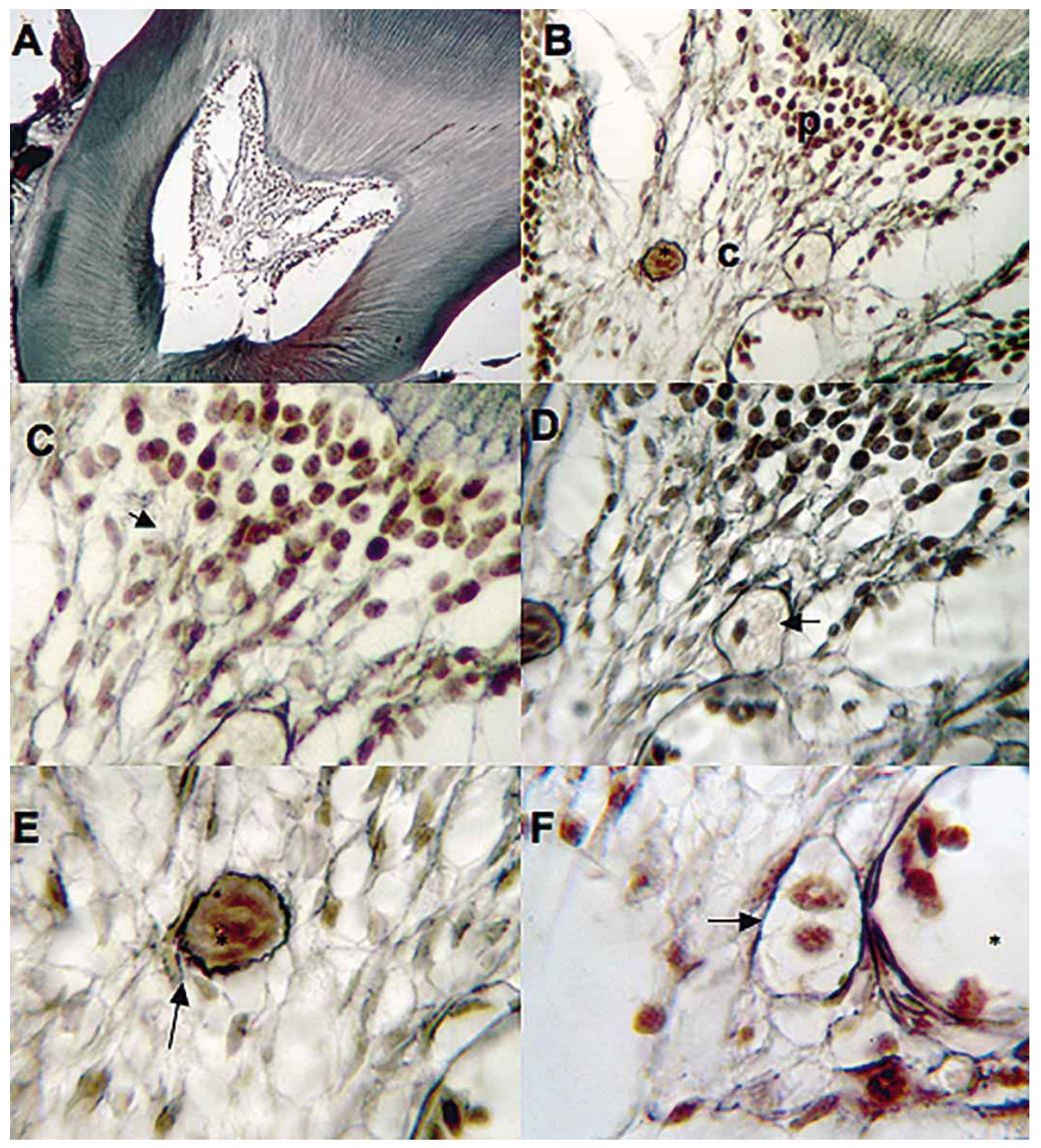

Fig. 3. Microfotografías de Cortes Histológicos de Molares de ratón con Técnica Reticular de Gomori. En A se observa la panorámica del tejido pulpar. En B se puede observar una alta densidad de fibras de fibras reticulares (teñidas en negro o gris), conformando el plexo reticular en la región central (c) dándole el sostén a los odontoblastos y periférica de la pulpa ( $p)$; el asterisco denota la presencia de vasos sanguíneos. La macrofotografía $\mathrm{C}$ permite ver un aumento del plexo (Flecha) en la región periférica y $D$ en la región central de la pulpa. Nótese en $E$ y $F$ que en la proximidad de los vasos sanguíneos $\left(^{*}\right)$ se observan fibras de Colágeno Tipo III bien desarrolladas (flechas). Aumento original de $4 \mathrm{X}$ en $\mathrm{A}, 10 \mathrm{X}$ en $B, 40 X$ en $C$ y D y $100 X$ en $E$ y F. 


\section{DISCUSIÓN}

Se identificaron las características histológicas mediante tinciones de H\&E, Tricrómica de Masson y tinción de Gomori para fibras reticulares del órgano dentinopulpar en ratones de 4 y 12 semanas. El tejido pulpar se encontró constituido por tejido conectivo laxo caracterizado por presentar una elevada celularidad. Histotopográficamente la pulpa dental ha sido dividida en cuatro regiones: odontoblástica, subodontoblástica, rica en células y central de la pulpa (Goldberg \& Smith). La naturaleza de las cuatro regiones topográficas es identificada con mayor claridad en órganos dentinopulpares de seres humanos (Goldberg \& Smith; Arana-Chavez \& Massa). Sin embargo, en este estudio, esta división clásica de la pulpa es de difícil reconocimiento ya que sólo se pudieron diferenciar dos zonas topográficas denominadas zona central de la pulpa y zona periférica de la pulpa. Estos hallazgos coinciden con las descripciones reportadas en el estudio clásico de la histología de la pulpa del ratón presentadas por Pinzón et al. en 1967, quienes determinaron que en mamíferos inferiores, la división topográfica descrita para la pulpa dental humana no tiene aplicación. Sin embargo, Pinzón et al. también plantearon la equivalencia de cada una de estas regiones con la pulpa humana. Así, la región central de la pulpa del ratón corresponde a las regiones rica en células y central descrita en los seres humanos, y la región periférica de la pulpa en ratón, corresponde a las regiones odontoblástica y subodontoblástica descritas en seres humanos.

La importancia de comprender la organización histotopográfica radica en la posibilidad de correlacionar las regiones con los diferentes tipos celulares presentes en la pulpa dental (Goldberg \& Lasfargues; Goldberg \& Smith; Arana-Chavez \& Massa; Angelova et al.), ya que estos autores han planteado la existencia de una relación entre las diferentes regiones con los grupos celulares ubicados en cada una de ellas. Los odontoblastos son las células dominantes en la región odontoblástica humana (Arana-Chavez \& Massa; Ruch et al., 1995); y en este estudio fueron encontrados en la región periférica de la pulpa en contacto con la matriz de predestina. Se identificaron como células cilíndricas, distribuidas en forma de empalizada con su núcleo polarizado lejos de la interfase pulpo-dentinal y con presencia de prolongación odontoblástica. Estos hallazgos se encuentran en congruencia con las descripciones de los odontoblastos realizadas por diferentes trabajos publicados (Goldberg \& Lasfargues; Goldberg \& Smith; Arana-Chavez \& Massa; Ruch et al.; Yu \& Abbott, 2007).
En la región central de la pulpa se hallaron abundantes células compatibles con fibroblastos pulpares, abundantes vasos sanguíneos y numerosas terminaciones nerviosas, también en concordancia con descripciones previas. El estudio no tuvo en cuenta las diferencias en la distribución de los diferentes tipos celulares encontrados entre los dos grupos de edad (4 y 12 semanas). Esto se debe en parte a que el diseño metodológico del presente estudio no consideró la valoración de las proporciones celulares ni estudios de recuento celular. Sin embargo, Pinzón et al. describieron una disminución progresiva en el número total de células en la pulpa de ratones; es necesario en estudios posteriores sobre ésta área analizar cuantitativamente la presencia celular para establecer su ritmo de variación celular asociado a los procesos de maduración tisular.

A nivel de la matriz extracelular fibrilar, este estudio evaluó la presencia y distribución de las fibras de colágeno y reticulares en el pulpa dental. Se encontraron diferencias en la distribución por edad y topográfica de dichas fibras. La presencia de colágeno se halló más abundante en la región central de la pulpa y realmente escasa en la región periférica de la pulpa (Abrahão et al., 2006). Estas diferencias fueron mucho más marcadas al identificar las variaciones con respecto a la edad. Así, se identificó un aumento en la presencia de fibras de colágeno en la región central de la pulpa en los ratones de 12 semanas al compararlos con los de 4 semanas. Estos hallazgos son congruentes con los reportados por Pinzón et al. en ratas Sprague-Dawley de 10, $15,30,60,90,125$ y 400 días de edad y con la asociación al proceso de envejecimiento pulpar (Schofield \& Weightman; Morse, 1991).

Un hallazgo controversial con los estudios de Pinzón et al. corresponde a la presencia de fibras reticulares. El presente estudio encontró una presencia casi constante de fibras reticulares en la región periférica de la pulpa tanto a las 4 como a las 12 semanas de edad; en tanto que la presencia en la región central de la pulpa disminuyó en los mismos rangos de edad, manteniéndose constante en la proximidad de los vasos sanguíneos. En el estudio de Pinzón et al. realizado con ratas Sprague-Dawley se encontró una disminución pareja de las fibras reticulares en todas las regiones de la pulpa de ratón a las diversas edades; quizás la razón de esta diferencia en la presencia de colágeno III pueda deberse a la utilización de cepas de ratones diferentes. 


\section{CONCLUSIONES}

La pulpa de ratones Albino Suizo no mostró la división típica en zona odontoblástica, zona subodontoblástica, zona rica en células y zona central de la pulpa; en su lugar se encontraron dos zonas: central y periférica con grupos poblacionales bien definidos.

No se encontraron diferencias entre los dos grupos de edad en las poblaciones celulares, sin embargo se encontró diferencia en la mayor presencia de colágeno en la pulpa de los ratones de 12 semanas.

ORTIZ, S. M. A. \& SALAZAR, M. L. Histological features of dental pulp in 4 and 12 week-old mice. Int. J. Odontostomat., 8(2):159-164, 2014.

ABSTRACT: Under physiological conditions, the dentinopulpar complex is not associated with remodeling and resorption. Histological findings show that the pulp as a connective tissue, has special features that bear on its ability to adapt. The purpose of this study was to evaluate the histological features of the dental pulp of mice of albino Swiss strain of 4 and 12 weeks-old. Eight hemimandibles of Swiss albino mice of 4 and 12 weeks-old were used. Hemimandibles were fixed in $4 \%$ paraformaldehyde, decalcified with EDTA $5 \%$ for 10 days at $4^{\circ} \mathrm{C}$ and included in Paraplast@; $5 \mathrm{~mm}$ sections were obtained and stained with H\&E, Masson's trichromic and silver impregnation. The histological characteristics of the pulp revealed two main areas in both age groups: the peripheral zone of the pulp formed by a set of cells compatible with odontoblastos and a poor-cell zone, and a central zone rich in pulp fibroblasts and blood vessels. Differences between age groups were seen in the expression of fibrillar collagen matrix. We found similarities in the presence of reticular fibers between the groups. The pulp of Swiss albino mice showed a division into two well-defined zones: central and peripheral. The greatest difference by age was in the amount of collagen.

KEY WORDS: dental pulp, odontoblasts, collagen.

\section{REFERENCIAS BIBLIOGRÁFICAS}

Abrahão, I. J.; Martins, M. D.; Katayama, E.; Antoniazzi, J. H.; Segmentilli, A. \& Margues, M. M. Collagen analysis in human tooth germ papillae. Braz. Dent. J., 17(3):208-12, 2006.

Angelova, A.; Takagi, Y.; Okiji, T.; Kaneko, T. \& Yamashita, Y. Immunocompetent cells in the pulp of human deciduous teeth. Arch. Oral Biol., 49(1):29-36, 2004.

Arana-Chavez, V. E. \& Massa, L. F. Odontoblasts: the cells forming and maintaining dentine. Int. J. Biochem. Cell Biol., 36(8):1367-73, 2004.

Goldberg, M. \& Lasfargues, J. J. Pulpo-dentinal complex revisited. J. Dent., 23(1):15-20, 1995.

Goldberg, M. \& Smith, A. J. Cells and extracellular matrices of dentin and pulp: a biological basis for repair and tissue engineering. Crit. Rev. Oral Biol. Med., 15(1):13-27, 2004.

Morse, D. R. Age-related changes of the dental pulp complex and their relationship to systemic aging. Oral Surg. Oral Med. Oral Pathol., 72(6):721-45, 1991.

Okiji, T. Pulp as a Connective Tissue. En: Hargreaves, K. \& Goodis, H. E. (Eds.). Seltzer and Bender's Dental Pulp. Carol Stream, Quintessence Publishing Co., 2002. pp.95122.
Pinzón, R. D.; Kozlov, M. \& Burch, W. P. Histology of rat molar pulp at different ages. J. Dent. Res., 46 (1):202-8, 1967.

Renard, E.; Lopez-Cazaux, S.; Guicheux, J.; Weiss, P.; Laboux, O. \& Alliot-Licht, B. Stem cells of dental pulp. C. R. Biol., 330(9):635-43, 2007.

Ruch, J. V.; Lesot, H. \& Bègue-Kirn, C. Odontoblast differentiation. Int. J. Dev. Biol., 39(1):51-68, 1995.

Schofield, J. D. \& Weightman, B. New knowledge of connective tissue ageing. J. Clin. Pathol. Suppl. (R. Coll. Pathol.), 12:174-90, 1978.

Yu, C. \& Abbott, P. V. An overview of the dental pulp: its functions and responses to injury. Aust. Dent. J., 52(1 Suppl.):S416, 2007.

Dirección para Correspondencia:

Mario Alejandro Ortiz Salazar

Odontólogo, Maestría en Ciencias Básicas Médicas. Especialista en Ortodoncia

Profesor Asistente, Departamento de Morfología Universidad del Valle, Campus San Fernando, Cali COLOMBIA

Email: mariortiz@yahoo.com

Recibido : 04-04-2014

Aceptado: 06-06-2014 